\title{
New species and synonymies in the genus Carrbotus Thorell, 1891 from India (Aranei: Salticidae: Salticini)
}

\author{
Новые виды и синонимы в роде Carrbotus Thorell, 1891 из \\ Индии (Aranei: Salticidae: Salticini)
}

\author{
John T.D. Caleb*, Chandan Bera, Shelley Acharya \\ Алкон Т.А. Калеб*, Чандан Бера, Шемли Ачарья
}

\author{
Zoological Survey of India, Prani Vigyan Bhawan, M-Block, New Alipore, Kolkata - 700 053, West Bengal, India. \\ E-mail: caleb87woodgate@gmail.com \\ * Corresponding author.
}

KEY WORDS: Andhra Pradesh, Araneae, Assam, Balochistan, China, Eastern Ghats, jumping spider, new combination, Tamil Nadu, taxonomy.

KEY WORDS: Андхра Прадеш, Araneae, Ассам, Балуджистан, Китай, Восточные Гаты, паук-скакунчик, новая комбинация, Тамилнад, таксономия.

ABSTRACT. Three new species of the genus Carrhotus Thorell, $1891-C$. andhra Caleb sp.n. $\left(\sigma^{7}\right), C$. assam Caleb sp.n. $\left(\sigma^{7}\right)$ and $C$. silanthi Caleb sp.n. $\left(O^{7}+\right)$ - are diagnosed and described from Andhra Pradesh, Assam and Tamil Nadu States of India respectively. A new combination is established, Carrhotus yunnanensis (Song, 1991) comb.n., ex Ptocasius. Five new synonyms have been proposed: Bianor piratus Sen, Dhali, Saha et Raychaudhuri, 2015 syn.n. with Carrhotus sannio Thorell, 1877; Carrhotus kevinlii Cao et Li, 2016 syn.n. with C. yunnanensis (Song, 1991); Marpissa decorata Tikader, 1974 syn.n., M. tikaderi Biswas, 1984 syn.n. and M. lakshmikantapurensis Majumder, 2004 syn.n. with Carrhotus viduus (C.L. Koch, 1846). C. viduus is recorded from Balochistan for the first time.

How to cite this article: Caleb J.T.D., Bera Ch., Acharya Sh. 2020. New species and synonymies in the genus Carrhotus Thorell, 1891 from India (Aranei: Salticidae: Salticini) // Arthropoda Selecta. Vol.29. No.1. P.51-66. doi: 10.15298/arthsel. 29.1.04

РЕЗЮМЕ. Диагностированы и описаны три новых вида из рода Carrhotus Thorell, $1891-C$. andhra Caleb sp.n. $\left(\sigma^{7}\right), C$. assam Caleb sp.n. $\left(\sigma^{7}\right)$ и $C$. silanthi Caleb sp.n. ( $\sigma^{7}$ ) — из индийских штатов Анхра Прадеш, Ассам и Тамил Наду. Предложена новая комбинация, Carrhotus yunnanensis (Song, 1991) comb.n., ех Ptocasius. Установлено пять новых синонимов: Bianor piratus Sen, Dhali, Saha et Raychaudhuri, 2015 syn.n. c Carrhotus sannio Thorell, 1877; Carrhotus kevinlii Cao et Li, 2016 syn.n. c $C$. yunnanensis (Song, 1991); Marpissa decorata Tikader, 1974 syn.n., M. tikaderi Biswas, 1984 syn.n. и M. lakshmikantapurensis Majumder, 2004 syn.n. с Carrhotus viduus (C.L. Koch, 1846). C. viduus впервые отмечен из Белуджистана.

\section{Introduction}

The genus Carrhotus was established by Thorell in 1891, with Plexippus viduus C. L. Koch, 1846 being its type species. The genus was earlier classified in the Hylleae by Simon [1901] and later considered a Chrysillinae taxon reasoning from the conformation of its copulatory organs [Prószyñski, 1976, 2017]. Based on both molecular and morphological evidence, the genus has recently been placed in the tribe Salticini of the subfamily Salticinae [Maddison, 2015]. Currently, the genus consists of 30 species worldwide [WSC, 2019], of which three species - C. sannio (Thorell, 1877), $C$. tristis Thorell, 1895 and C. viduus (C.L. Koch, 1846) are known from India [WSC, 2019]. The aims of the present paper are: (1) to describe three new Carrhotus species: C. andhra sp.n. $\left(O^{7}\right)$ from the Eastern Ghats, Andhra Pradesh, C. assam sp.n. $\left(O^{7}\right)$ from the rainforest of the Dehing Patkai Wildlife Sanctuary, Assam and $C$. silanthi Caleb sp.n. $\left(\sigma^{7}+\right.$ ) from the scrub jungle region of Tambaram, Tamil Nadu; (2) to provide new distributional data on C. viduus (C.L. Koch, 1846) in the Indian fauna; and (3) to propose a new combination and five new synonymies.

\section{Material and methods}

Morphological examination of specimens was carried out under a Leica EZ4 HD stereomicroscope. Detailed micro-photographs were acquired by means of a Leica M205A stereomicroscope equipped with a Leica DFC500 HD camera enabled with a Leica Application Suite (LAS) version 3.8. The epigyne was dissected and cleared in $10 \% \mathrm{KOH}$. Temporary slide preparations were photographed by means of a Leica DM1000 compound microscope equipped with a Leica EC3 camera. A Zeiss EVO 18 Scanning Electron Microscope was used to study and obtain detailed morpho- 

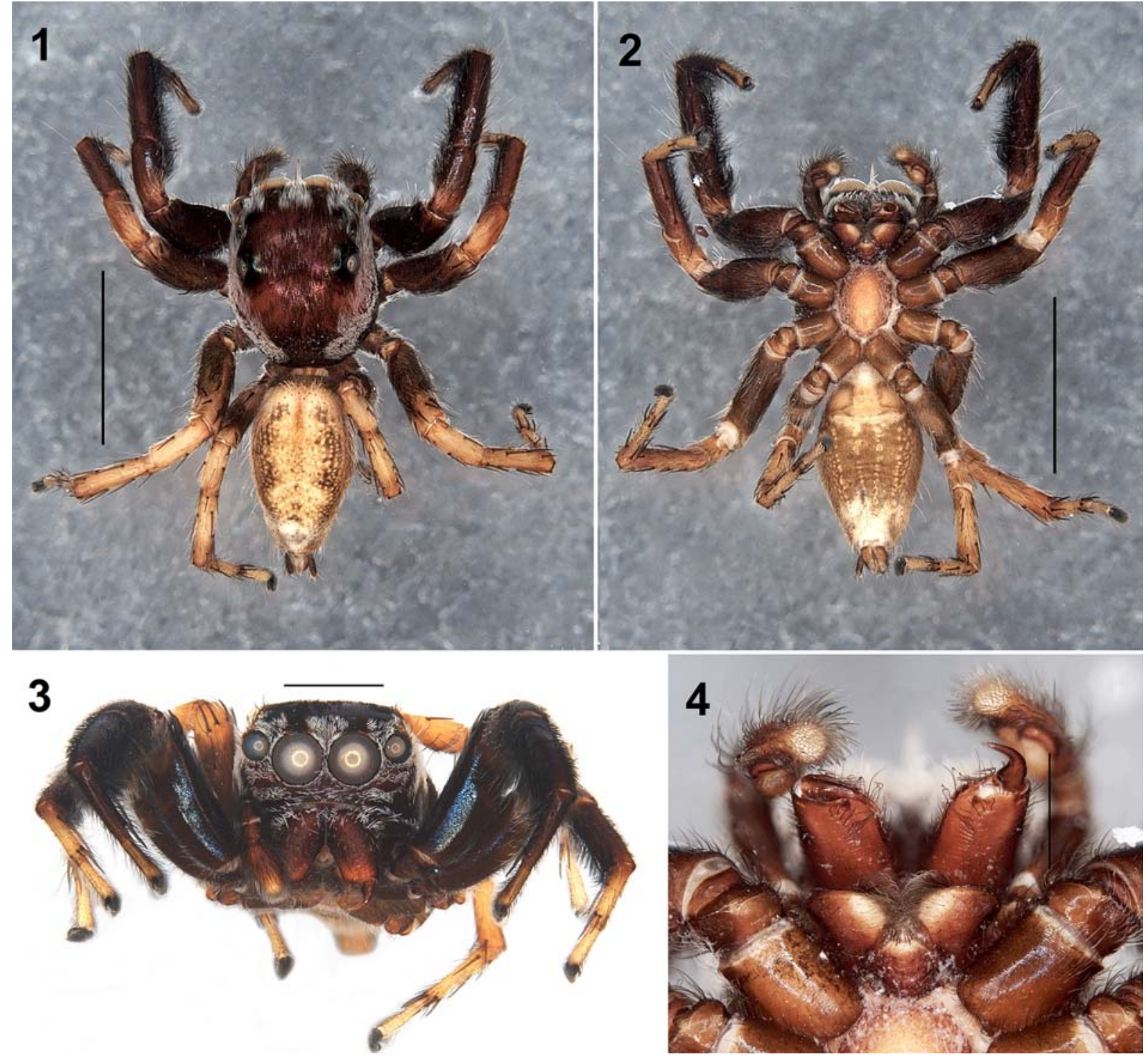

Figs 1-4. Somatic morphology of Carrhotus andhra Caleb sp.n., holotype male: 1 - general appearance, dorsal view; 2 - ditto, ventral view; 3 - ditto, front view; 4 - chelicerae, ventral view. Scale bars: (1-2) $2 \mathrm{~mm}$, (3) $1 \mathrm{~mm}$, (4) $0.5 \mathrm{~mm}$.

Рис. 1-4. Соматическая морфология Carrhotus andhra Caleb sp.n., голотип самец: 1 - общий вид, сверху; 2 - тоже, снизу; 3 тоже, спереди; 4 - хелицеры, снизу. Масштаб: (1-2) 2 мм, (3) 1 мм, (4) 0,5 мм.

logical images. Line drawings were prepared by using the GNU Image Manipulation Program [Montesanto, 2015]. Distribution maps were prepared by using the online mapping software SimpleMappr [Shorthouse, 2010]. Where locality coordinates were not available from data labels, they were approximated using Google Earth. Leg measurements are given in the following order: total (femur, patella, tibia, metatarsus, tarsus). Spine positions are as follows: prolateral, dorsal, retrolateral and ventral. All measurements are in $\mathrm{mm}$. The type material is kept in the National Zoological Collections, Zoological Survey of India (NZC-ZSI), Kolkata (erstwhile Calcutta).

Abbreviations used in the text: AER - anterior eye row; ALE - anterior lateral eye; AME - anterior median eye; D — described; EFL — eye field length; PER — posterior eye row; PLE - posterior lateral eye; PME - posterior median eye; RTA — retrolateral tibial apophysis; NZC-ZSI -
National Zoological Collections, Zoological Survey of India, Kolkata.

\section{Provisional key to the Indian CarRHotus Species}

1. Males 2

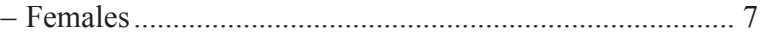

2. Tegulum with a prolateral, knife-like protrusion (arrowed in Fig. 9), RTA bifurcated at its tip (Fig. 10) C. andhra sp.n.

- Tegulum without a prolateral protrusion, RTA pointed .3

3. Embolus filiform, longer than cymbium (figs 8, 9 in Prószyński [1992])

C. tristis

- Embolus otherwise (Figs 20, 23, 65) …………….......... 4

4. Embolus hook-shaped (Figs 20, 23); RTA straight, directed anteriad (Figs 21, 24)... 

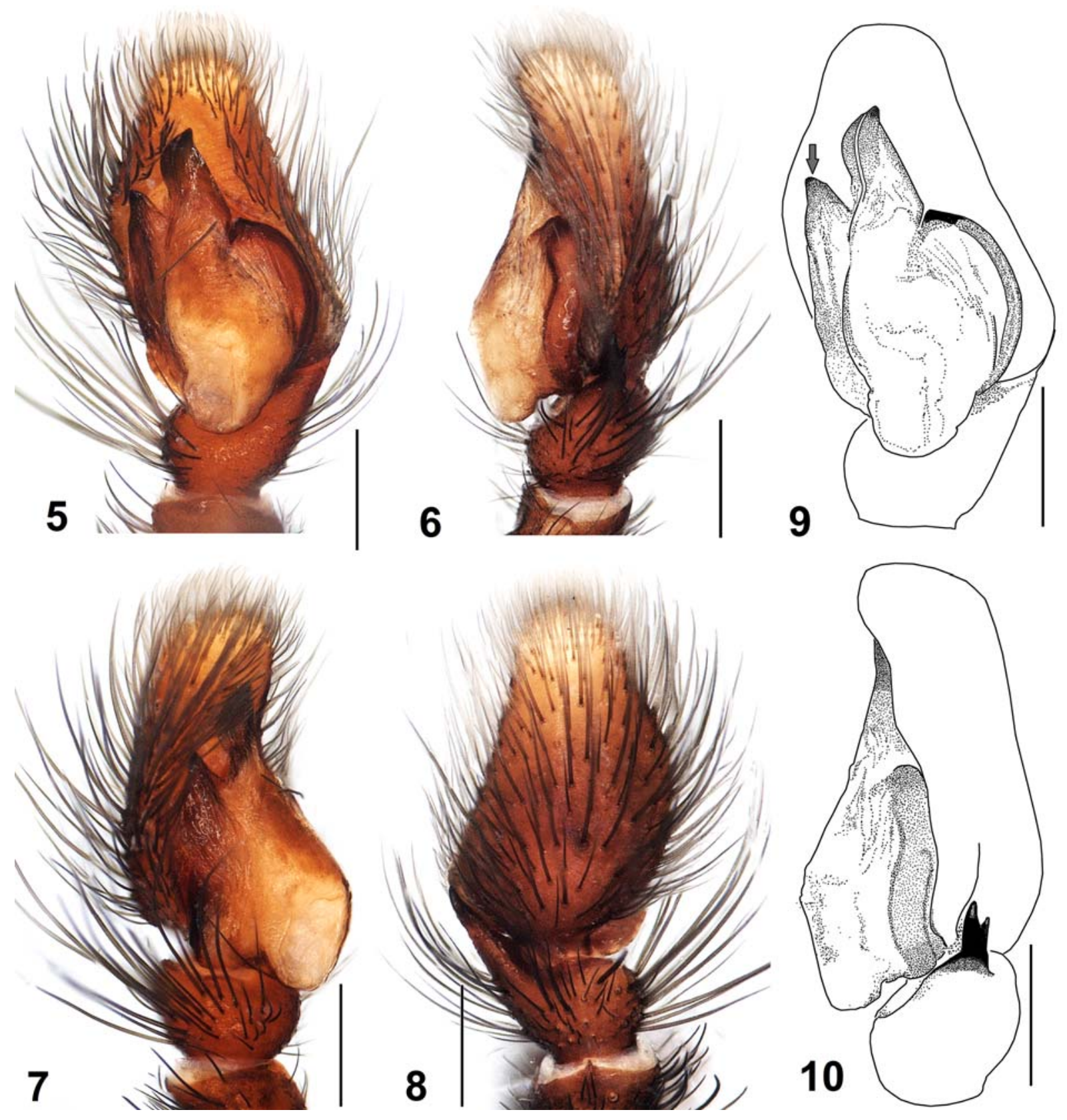

Figs 5-10. Left male palp of Carrhotus andhra Caleb sp.n., holotype: 5, 9- ventral view; 6,10 - ditto, retrolateral view; 7 - ditto, prolateral view; 8 - ditto, dorsal view. Scale bars: $0.2 \mathrm{~mm}$.

Рис. 5-10. Левая пальпа самца Carrhotus andhra Caleb sp.n., голотип: 5 - снизу; 6 - сбоку-сзади; 7 - сбоку-спереди; 8 сверху. Масштаб: 0,2 мм.

- Embolus straight and thick, conical apically (Figs 48, 65); RTA hook-shaped (Fig. 49) or at least bent ventrad (Fig. 66)

5. Dorsum brownish, without a white colour pattern (Fig. 11); male palp (Figs 20,21) C. assam sp.n.

- Dorsum dark brown, with two pairs of white spots (Fig. 22); male palp (Figs 23, 24)

C. sannio

6. RTA markedly hook-shaped, bent ventrad (Figs 45, 49)
- RTA only slightly bent ventro-apicad (Figs 62, 66)

C. viduus

7. Copulatory openings placed anteriorly (Figs 63, 71, 73, 81) C. viduus

- Copulatory openings placed posteriorly (Figs 26, 46, 50)

8. Insemination ducts comparatively long and loop anteriorly (Fig. 27) ..................................................... C. sannio - Insemination ducts comparatively short and diverge laterally (Figs 47,51$)$... C. silanthi sp.n. 


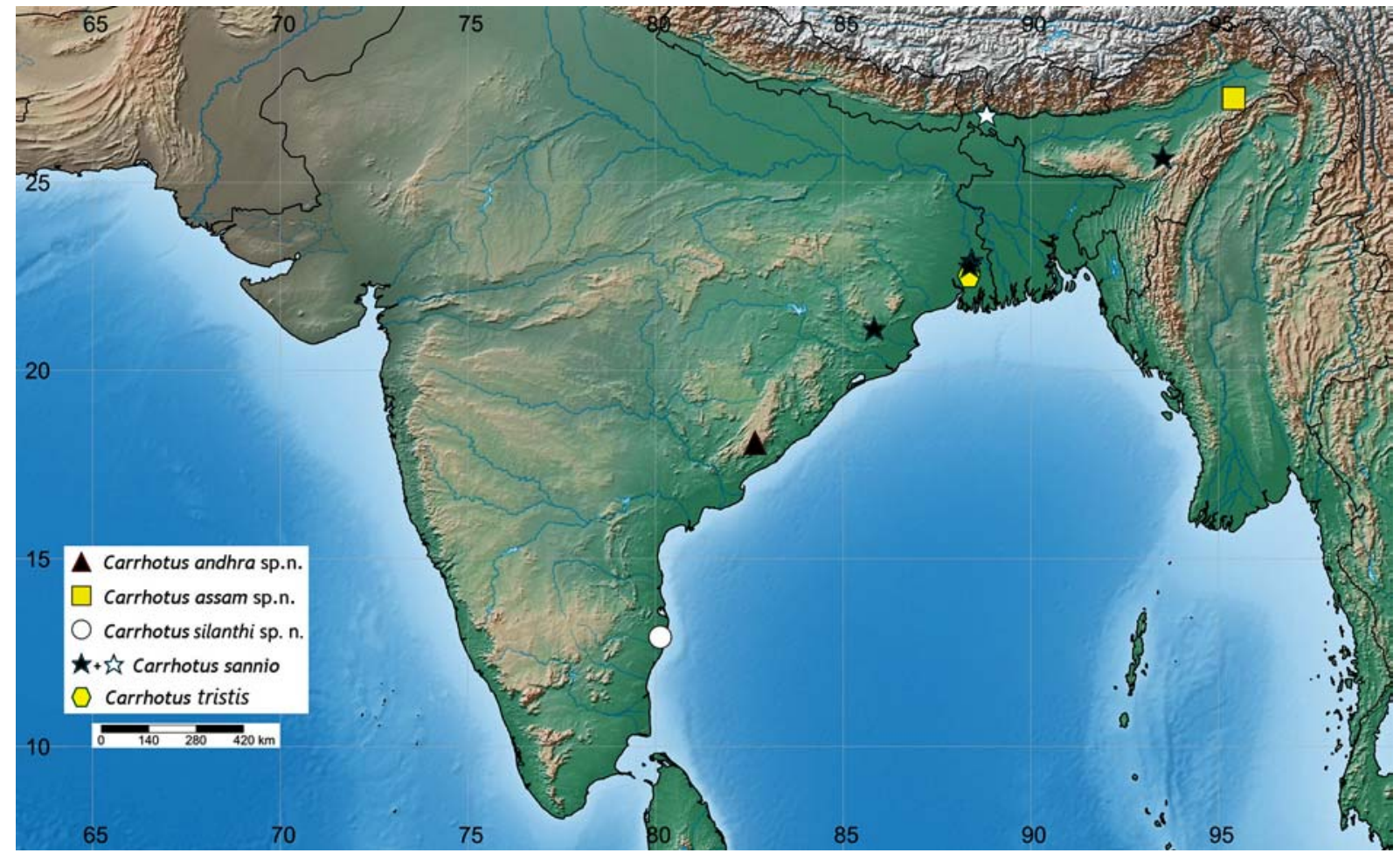

Map 1. Collecting localities of five Indian Carrhotus species. White star represents the type locality of Bianor piratus (= Carrhotus sannio).

Карта 1. Точкии находок пяти индийских видов Carrhotus. Белая звездочка отмечает типовой локалитет Bianor piratus (= Carrhotus sannio).

\section{Survey of species}

\section{Carrhotus andhra Caleb, sp.n.}

Figs 1-10, Map 1.

TYPE. Holotype $\sigma^{7}$ (NZC-ZSI 6733/18) from India, Andhra Pradesh, Visakhapatnam Distr., Paderu $\left(18.08^{\circ} \mathrm{N}, 82.65^{\circ} \mathrm{E}\right), 1015$ m a.s.1., 8.09.2018, P. Sardar \& G.M. Koley.

ETYMOLOGY. The species is named after the Indian state, Andhra Pradesh from where the holotype was collected. The name is a noun in apposition.

DIAGNOSIS. The species can be distinguished from all its congeners by the presence of a prolateral, knife-like protrusion of the tegulum (arrowed in Fig. 9) and the RTA bifurcated at its tip (Figs 6, 10).

DISTRIBUTION. India (Andhra Pradesh) (Map 1).

DESCRIPTION. MALE. Total length: 4.19; carapace: 2.11 long, 1.76 wide; abdomen: 2.07 long, 1.21 wide. Carapace brownish, with three patches of white hairs along the anterior margin; broad white patch of hairs present on lateral sides of the carapace (Fig. 1). Anterior eyes surrounded by white orbital setae; clypeus sparsely covered with white scales (Fig. 3). Eye measurements: AME 0.53, ALE 0.30, PME 0.06, PLE 0.24, AER 1.73, PER 1.48, EFL 0.94. Clypeus height 0.16. Sternum oval, yellowish brown. Chelicerae brown, with two promarginal and one retromarginal teeth. Labium and maxillae yellow-brown (Fig. 4). Legs brownish, with light brown patellae; metatarsi II and tarsi II yellowish; tibiae, metatarsi and tarsi of legs III \& IV yellowish (Figs 1, 2). Leg measurements: I 6.11 (1.87, 1.10, 1.47, $1.00,0.67)$; II 5.03 (1.64, 0.96, 1.07, 0.81, 0.55); III 6.05
$(2.12,0.99,1.07,1.15,0.72) ;$ IV $5.44(1.73,0.71,1.10$ $1.18,0.72)$. Leg formula: 1342 . Leg spination: femora I 0500, II 0700, III 0700, IV 0700; patellae I 2010, II-IV 1010; tibiae I 2006, II 3026, III 4143, IV 4143; metatarsi I 1004, II 1024, III 3034, IV 4043; tarsi I-IV 0000. Abdomen yellow-brown, with a pair of lateral brown longitudinal patches; mid-dorsum with chevron-shaped markings (Fig. 1); venter brownish. Spinnerets brownish (Fig. 1). Palps brownish; cymbium with a retromarginal protrusion completely covering the RTA in ventral view; embolus thick, pointing apically; RTA thick, with a bifid tip, directed vertically (Figs 5-10).

Female unknown.

\section{Carrhotus assam Caleb, sp.n.}

Figs 11-17, 20, 21, Map 1.

TYPE. Holotype $\sigma^{7}$ (NZC-ZSI 6938/18) from India, Assam, Dehing Patkai Wildlife Sanctuary $\left(27.24^{\circ} \mathrm{N}, 95.41^{\circ} \mathrm{E}\right), 137 \mathrm{~m}$ a.s.1., 16.08.2016, T.K. Roy.

ETYMOLOGY. The species is named after the Indian state Assam from where the holotype was collected. The name is as a noun in apposition.

DIAGNOSIS. The species is closely related to $C$. yunnanensis (Song, 1991) comb.n., but can be distinguished from it by the following characters (cf. Figs 15, 16, 20, 21 with Figs 18, 19): the orientation of RTA which is directed vertically, at about 20 degrees, in both ventral and retrolateral views in $C$. assam sp.n., while it protrudes laterally, at about 45 degrees, in ventral view and directed ventrally in retrolateral view, in C. yunnanensis; palpal tibia twice as long as its width (thrice in C. yunnanensis); tibia is about 2/ 3 rd of the cymbial length (4/5th in C. yunnanensis). 

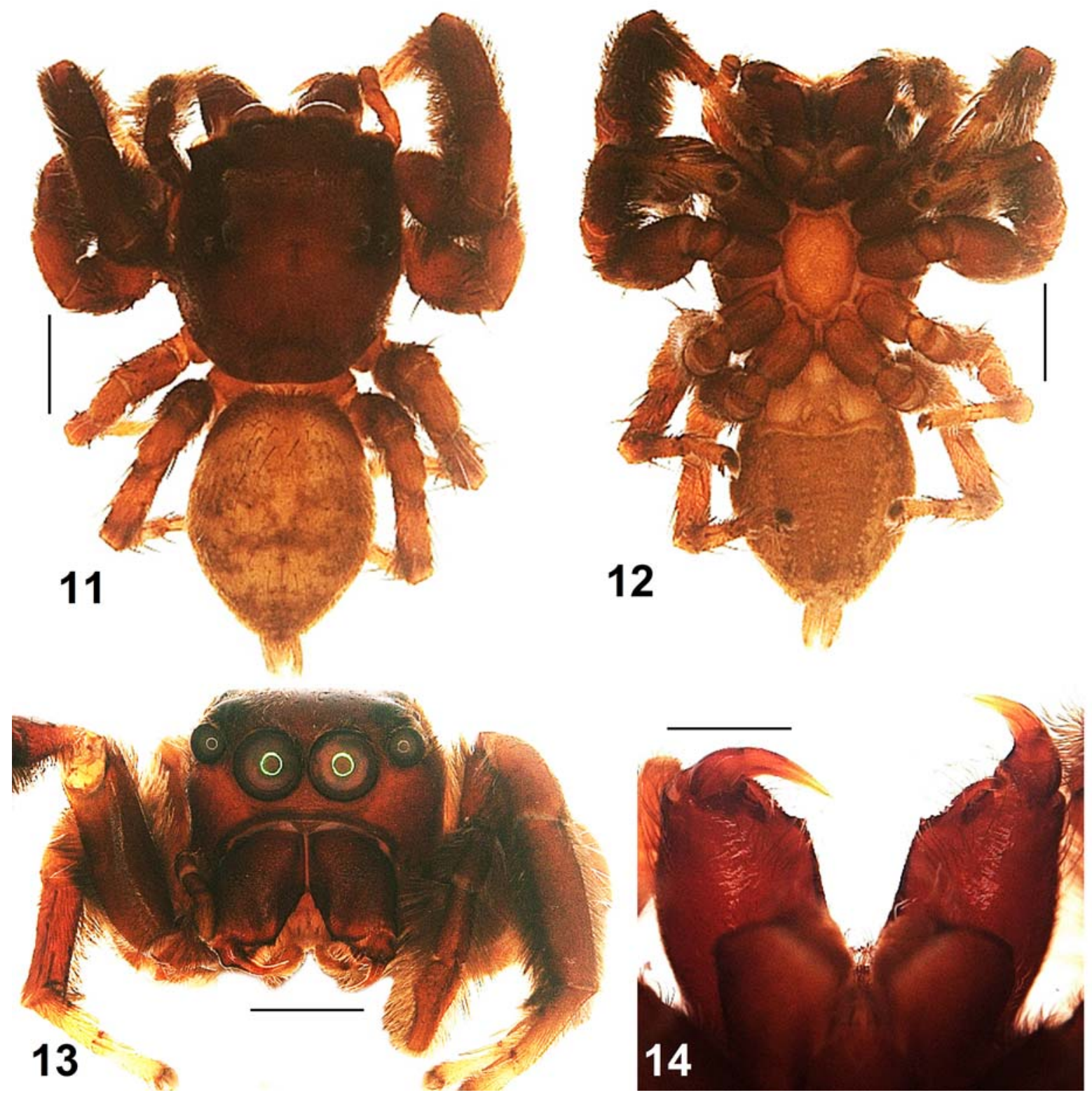

Figs 11-14. Somatic morphology of Carrhotus assam Caleb sp.n., holotype male: 11 - general appearance, dorsal view; 12 - ditto, ventral view; 13 - ditto, front view; 14 - chelicerae, ventral view. Scale bars: (1-3) $1 \mathrm{~mm}$, (4) $0.5 \mathrm{~mm}$.

Рис. 11-14. Соматическая морфология Carrhotus assam Caleb sp.n., голотип-самец: 11 - общий вид, сверху; 12 - тоже, снизу; 13 - тоже, спереди; 14 - хелицеры, снизу. Масштаб: (1-3) 1 мм, (4) 0,5 мм.

DISTRIBUTION. India (Assam) (Map 1).

DESCRIPTION. Male. Total length: 4.95; carapace: 2.55 long, 2.19 wide; abdomen: 2.40 long, 1.83 wide. Carapace reddish brown, posterior eyes surrounded by black (Fig. 11). Anterior eyes surrounded by pale white orbital setae; clypeus brown (Fig. 13). Eye measurements: AME 0.64, ALE 0.34, PME 0.10, PLE 0.37, AER 2.09, PER 2.44, EFL 1.54. Clypeus height 0.18. Sternum yellowish brown. Chelicerae brown, with two small promarginal and a large retromarginal teeth. Labium and maxillae brownish (Fig. 14). Legs brown with dark brown annulations (Figs 11, 12). Leg measurements: I $7.66(2.27,1.50,1.89,1.28,0.72)$; II
$6.01(1.85,1.22,1.27,1.06,0.61)$; III $6.55(2.16,1.13,1.20$, $1.29,0.77)$; IV $6.44(2.00,1.02,1.27,1.43,0.72)$. Leg formula: 1342. Leg spination: femora I-IV 0700; patellae IIV 1010; tibiae I 4026, II 4036, III 4043, IV 4043; metatarsi I 0004, II 1014, III 3034, IV 4044; tarsi I-IV 0000. Abdomen brownish, with dark brown patches (Fig. 11); venter brownish. Spinnerets yellow-brown (Fig. 12). Palps brownish; cymbium with an apical protrusion; embolus emerging at nine o'clock position, thin and needle-like, with a membrane; palpal tibia long; RTA thick, broad at its base and gently curving, pointed at its tip (Figs 15-17, 20, 21).

Female unknown. 


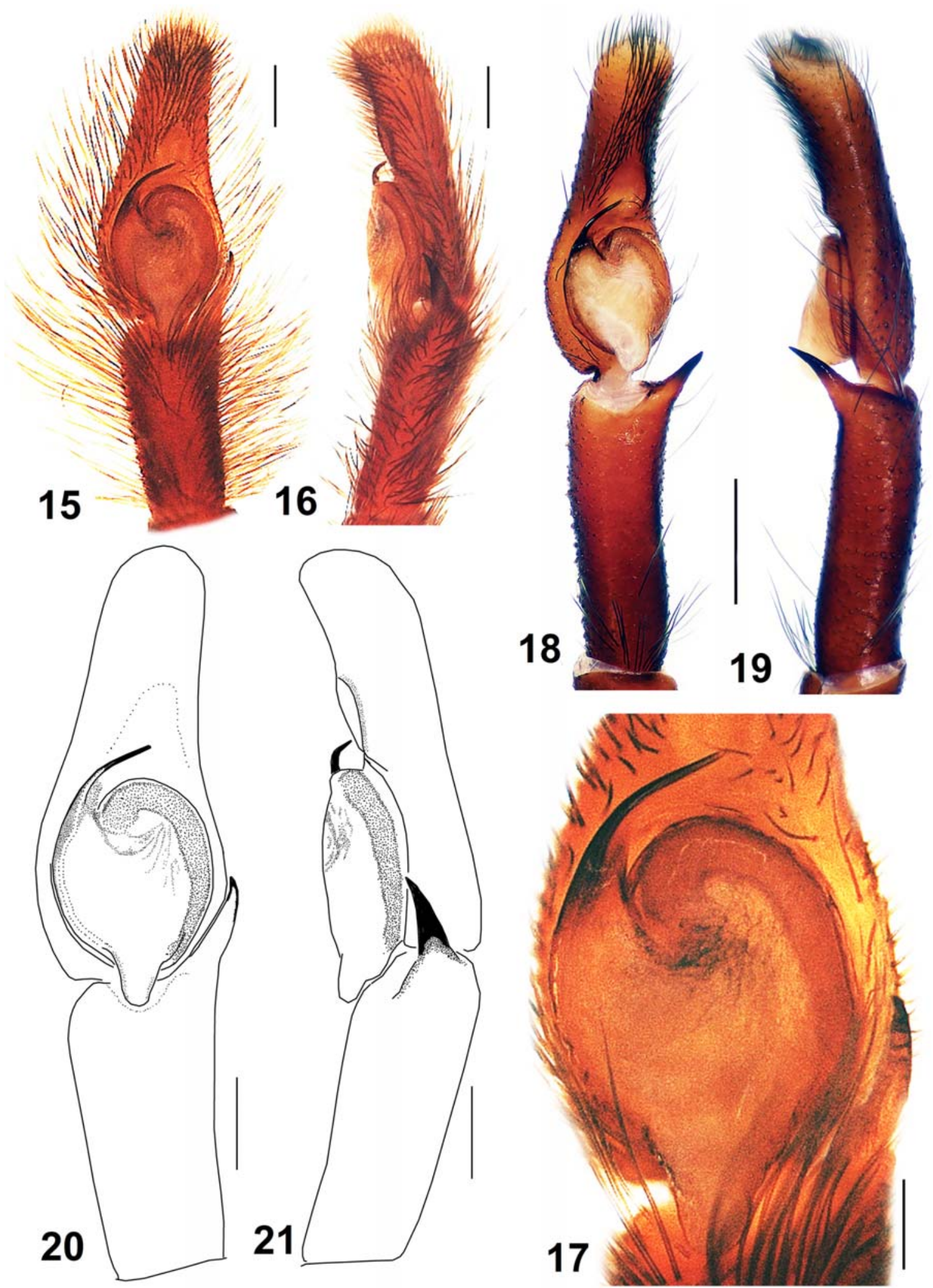

Figs 15-21. Left male palps of Carrhotus assam Caleb sp.n., holotype (15-17, 20, 21) and C. yunnanensis (Song, 1991) comb.n. (18, 19; from Cao et al. [2016], used under a Creative Commons Attribution 4.0 International License): 15, 17, 18, 20 — ventral view; 16, 19, 21 - retrolateral view. Scale bars: (17) $0.1 \mathrm{~mm},(15-16,20,21) 0.2 \mathrm{~mm},(18) 0.5 \mathrm{~mm}$.

Рис. 15-21. Левые пальпы самцов Carrhotus assam Caleb sp.n., голотип $(15-17,20,21)$ и C. yunnanensis (Song, 1991) comb.n. $(18,19 ;$ из Сао et al. [2016], используется в соответствии с международной лицензией Creative Commons Attribution 4.0): 15, 17, 18, 20 - снизу; 16, 19, 21 - сбоку-сзади. Масштаб: (17) 0,1 мм, (15-16, 20, 21) 0,2 мм, (18) 0,5 мм. 

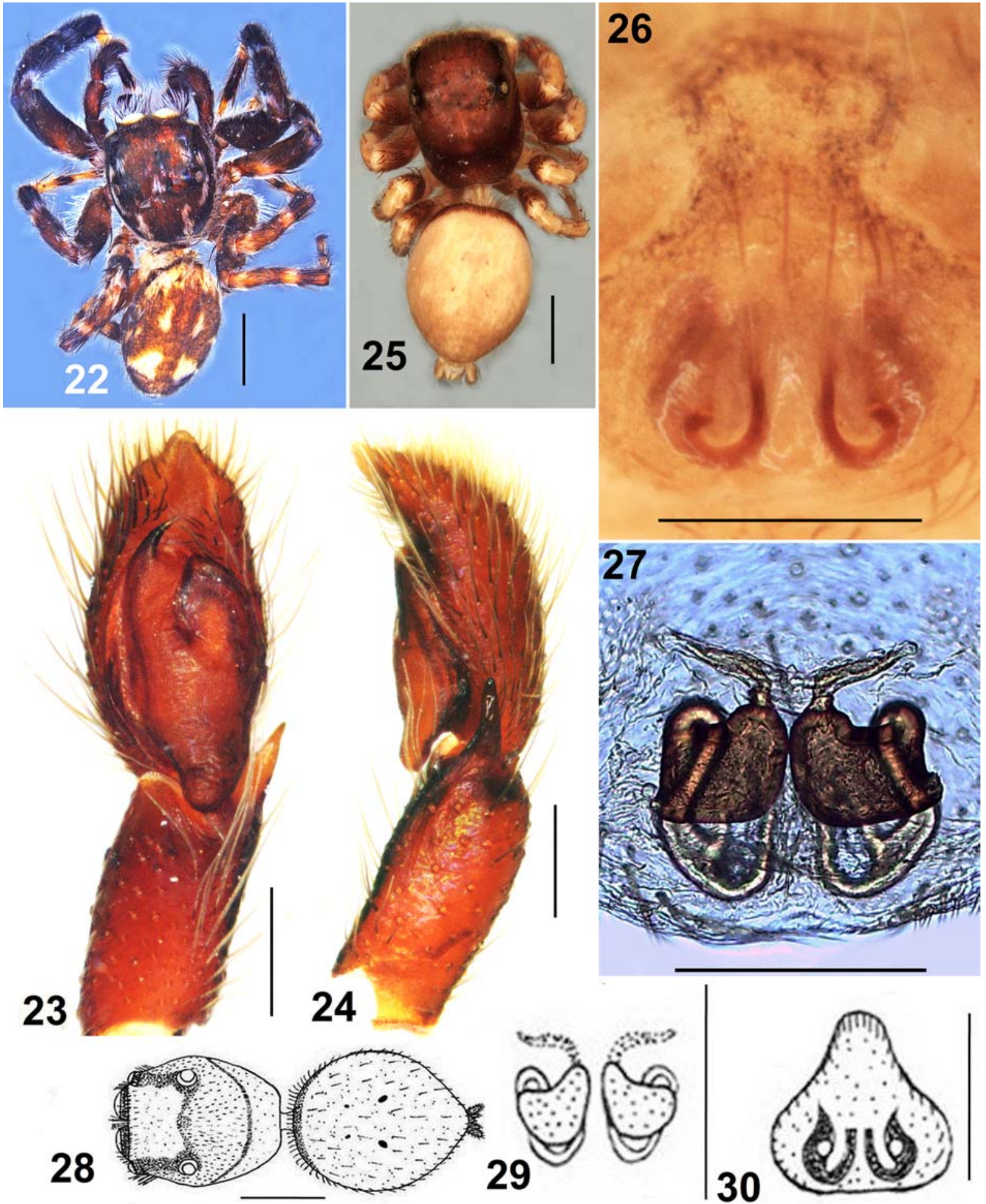

Figs 22-30 Somatic morphology and copulatory organs of Carrhotus sannio Thorell, 1877, male from Nagaland, female from West Bengal: 22, 25, 28 - general appearance, dorsal view; 23 - male left palp, ventral view; 24 - ditto, retrolateral view; 26, 30 - epigyne, ventral view; 27, 29 - vulva, dorsal view. Original illustrations of Bianor piratus (28-30) are from Sen et al. [2015]. Scale bars: (23, 24, $26,27) 0.2 \mathrm{~mm},(29,30) 0.25 \mathrm{~mm},(22,25,28) 1 \mathrm{~mm}$.

Рис. 22-30 Соматическая морфология и копулятивные органы Carrhotus sannio Thorell, 1877, самец из Нагаланда, самка из Западного Бенгала: 22, 25, 28 - общий вид, сверху; 23 - левая пальпа самца, снизу; 24 - тоже, сбоку-сзади; 26, 30 - эпигина, снизу; 27, 29 - вульва, сверху. Оригинальные иллюстрации Bianor piratus (28-30) из Sen et al. [2015]. Масштаб: $(23,24,26,27)$ 0,2 мм, $(29,30) 0,25$ мм, $(22,25,28) 1$ мм. 

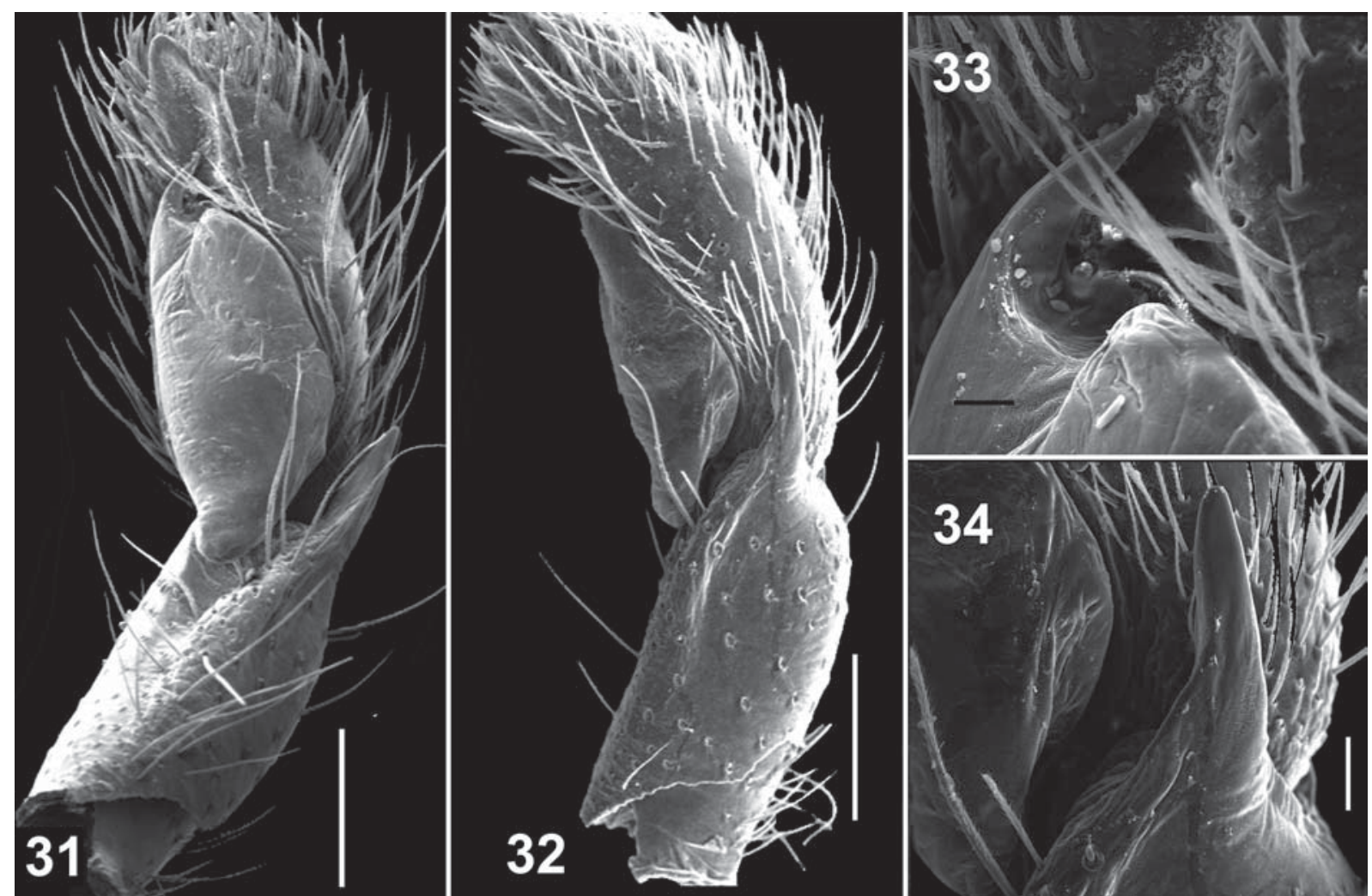

Figs 31-34. Left male palp of Carrhotus sannio Thorell, 1877, male from Nagaland: 31 - ventral view; 32 - retrolateral view; 33 embolus, ventral view; 34 - magnified view of RTA, retrolateral view. Scale bars: (33) $0.02 \mathrm{~mm}$, (34) $0.04 \mathrm{~mm},(31,32) 0.2 \mathrm{~mm}$.

Рис. 31-34. Левая пальпа самца Carrhotus sannio Thorell, 1877, самец из Нагаланда: 31 - снизу; 32 - сзади-сбоку; 33 эмболюс, снизу; 34 - отросток голени, сбоку-сзади. Масштаб: (33) 0,02 мм, (34) 0,04 мм, (31, 32) 0,2 мм.

\section{Carrhotus sannio Thorell, 1877}

Figs 22-34, Map 1.

Plexippus sannio Thorell, 1877: 617 (D $\left.\odot^{7}\right)$.

Carrhotus sannio: Żabka, 1985: 207, figs 63-70 ( $\left.\sigma^{7}+\right)$; Prószyński, 1992: 168, fig. $6\left(0^{7}\right)$; Jastrzębski, 1999: 3, figs 4-7 $\left(0^{7}\right)$.

Bianor piratus Sen, Dhali, Saha et Raychaudhuri, 2015: 35, figs 97-101, pl. 13 (D+); the type series in the Ramakrishna Mission Vivekananda University, Narendrapur, Kolkata; digital images of the paratype + examined; comb.n., syn.n.

Bianor piratus: Dhali et al., 2017: 32, figs 58-62, pl. 16 (+).

For a complete list of taxonomic references see WSC [2019].

MATERIAL. INDIA: 1 o (NZC-ZSI 6374/18), West Bengal, North 24 Parganas Distr., Halisahar $\left(22.924791^{\circ} \mathrm{N}, 88.42254^{\circ} \mathrm{E}\right)$, $17 \mathrm{~m}$ a.s.1., 21.01.2017, S. Talukdar; 1 \% (NZC-ZSI 6661/18), same state and distr., Palta $\left(22.78^{\circ} \mathrm{N}, 88.37^{\circ} \mathrm{E}\right), 12 \mathrm{~m}$ a.s.1., 29.10.1980, B. Biswas; $1 \sigma^{7}$ (NZC-ZSI 6400/18), Nagaland, Peren Distr., Intangki National Park $\left(25.663889^{\circ} \mathrm{N}, 93.512778^{\circ} \mathrm{E}\right), 195 \mathrm{~m}$ a.s.1., 24.03.2017, Swati \& Aneesh.

DISTRIBUTION. Réunion, India to Indonesia (Sulawesi) [WSC, 2019]; the distribution in India is shown on Map 1 .

COMMENTS. Bianor piratus was described from the holotype female and four paratype females collected from Dhupjhora, Gorumara National Park, West Bengal [Sen et al., 2015]. A detailed examination of the female specimen recently collected from West Bengal and its comparison with the images of presumably one of the paratypes provided to JC by Prof. Dinendra Raychaudhuri has revealed that B. piratus actually belongs to the genus Carrhotus. The epigyne with longitudinal depressions separated by a septum and the internal structures with long insemination ducts rising anteriorly and looping before entering the anterior region of the elongated spermathecae are identical to those of C. sannio (cf. Figs 28-30 with Figs 25, 26, 27 and figs 67-70 in Żabka [1985]). Thus it is safe to conclude that this species is to be a member of Carrhotus and be considered a junior synonym of $C$. sannio.

\section{Carrhotus silanthi Caleb sp.n.}

Figs 35-56, Map 1.

TYPES. Holotype $\sigma^{\top}$ (NZC-ZSI 6939/18) and paratype $q$ (NZCZSI 6940/18) from India, Chennai, Tambaram, Madras Christian College $\left(12.9141^{\circ} \mathrm{N}, 80.1233^{\circ} \mathrm{E}\right), 32 \mathrm{~m}$, a.s.1., 3.09.2016, J. Caleb \& J. Daniel.

ETYMOLOGY. The specific name is taken from Tamil word 'silanthi' meaning 'spider'. It is treated as a noun in apposition.

DIAGNOSIS. The males of $C$. silanthi sp.n. can be distinguished from those of C. viduus (C.L. Koch, 1846) by their body colour pattern with no longitudinal stripes on the carapace and abdomen (present in C. viduus) (cf. Figs 35, 36 with Figs 57, 59 and fig. 8 in Jastrzębski [1999] and fig. 1 in Caleb [2016]). The palpal conformation of C. silanthi sp.n. closely resembles that of $C$. viduus as well, but can be distinguished by the thinner embolus, the longer palpal tibia and the claw-shaped RTA (cf. Figs 44, 45, 52-55 with Figs $61,62,65-69)$. The females can be readily distinguished 


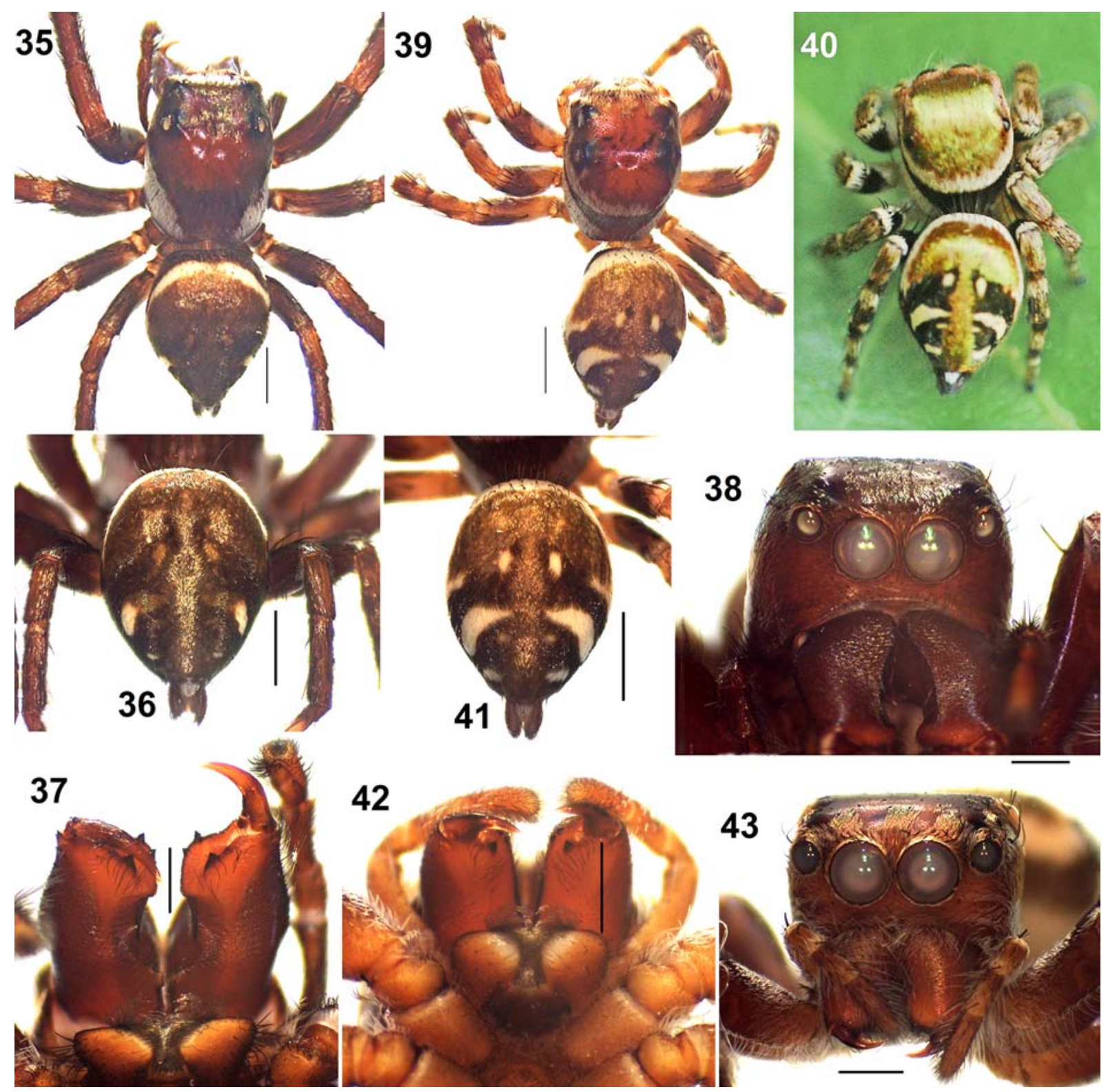

Figs 35-43. Somatic morphology of Carrhotus silanthi Caleb sp.n., holotype male (35-38), paratype female (39-43): 35, 39 - body, dorsal view; 36, 41 - abdomen, dorsal view; 37, 42 - chelicerae, ventral view; 38, 43 - carapace, front view; 40 - habitus, live specimen. Scale bars: $(35,36,39,41) 1 \mathrm{~mm} ;(37,38,42,43) 0.5 \mathrm{~mm}$.

Рис. 35-43. Соматическая морфология Carrhotus silanthi Caleb sp.n., голотип-самец (35-38), паратип-самка (39-43): 35, $39-$ тело, сверху; 36, 41 - брюшко, сверху; 37, 42 - хелицеры, снизу; 38, 43 - головогрудь, спереди; 40 - внешний вид живого экземпляра. Масштаб: $(35,36,39,41) 1$ мм; $(37,38,42,43) 0,5$ мм.

from those of $C$. viduus by the internal duct system: viz., the insemination ducts move posteriorly towards each other to the copulatory openings (Figs 47, 51) (ducts move anteriorly and almost sub-parallel in C. viduus, see Figs 64, 82).

DISTRIBUTION. India (Tamil Nadu) (Map 1).

DESCRIPTION. MALE (holotype). Total length: 6.14; carapace: 3.02 long, 2.37 wide; abdomen: 3.12 long, 2.31 wide. Carapace reddish brown, covered with iridescent hairs providing a metallic sheen (Fig. 35); lateral margins of carapace lined by broad patches of white hairs; posterior region black. Clypeal region brownish; eyes surrounded by yellowish orbital setae (Fig. 38). Eye measurements: AME 0.56,
ALE 0.33, PME 0.08, PLE 0.25, AER 1.91, PER 1.97, EFL 1.19. Clypeus height 0.16 . Chelicerae dark brown, with curved outer margins and excavated inner margins; two promarginal and one retromarginal teeth (Fig. 37). Sternum oval, brownish; labium and maxillae dark brown, with paler outer margins. Leg I robust; femora I-IV black dorsally; patellae and tibiae dark brown; tarsi and metatarsi of all legs brownish (Figs 35, 36). Leg measurements: I 8.12 (2.27, $1.46,2.03,1.49,0.87)$; II 6.30 (1.92, 1.11, 1.37, 1.18, 0.72); III $6.36(2.09,1.17,1.16,1.25,0.69)$; IV 6.01 (1.99, 0.96, $1.16,1.28,0.62)$. Leg formula: 1324 . Leg spination: femora I 0600, II 0700, III 0700, IV 0700; patellae I-IV 1010; 


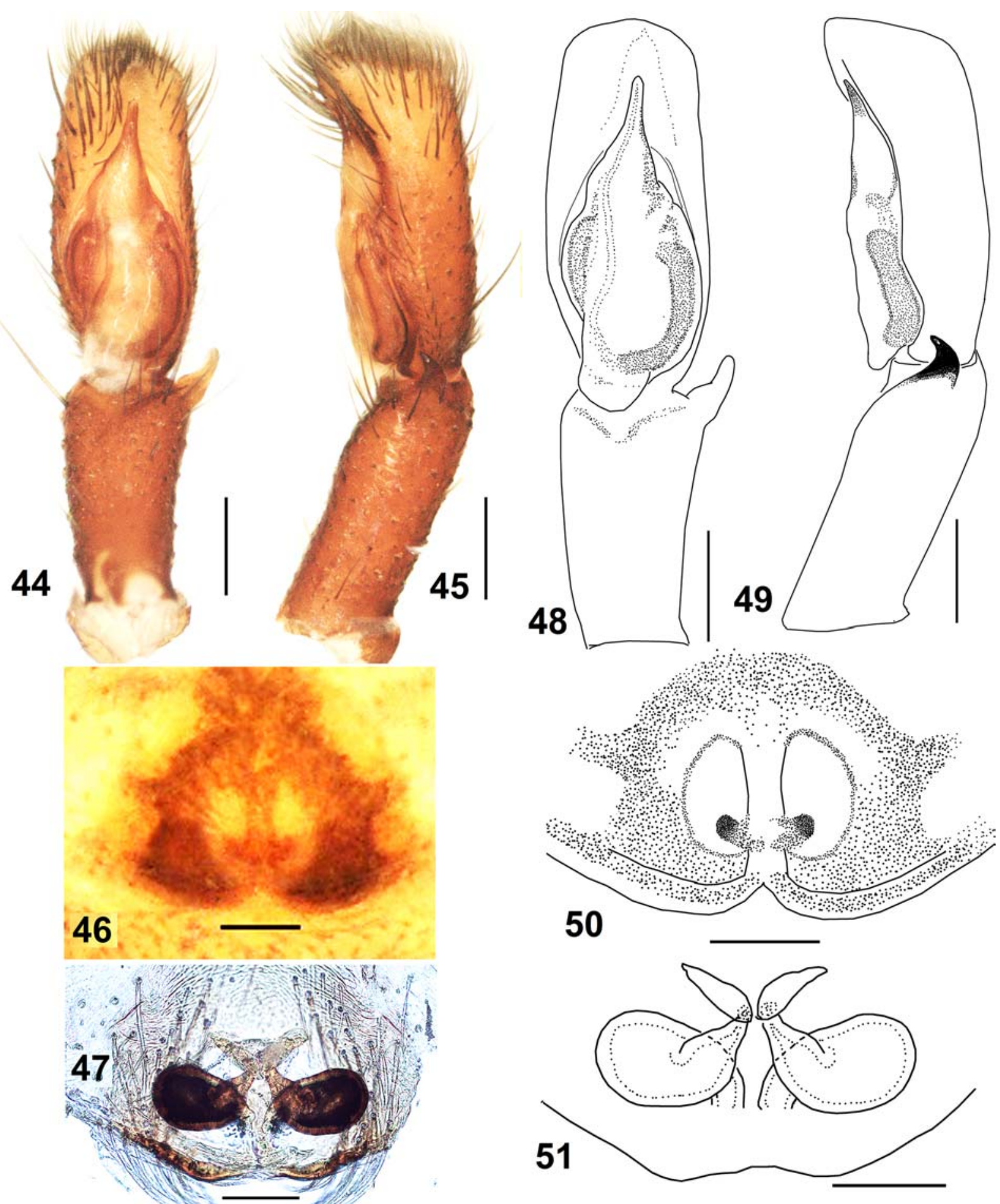

Figs 44-51. Copulatory organs of Carrhotus silanthi Caleb sp.n., holotype male and paratype female: 44,48 - left male palp, ventral view; 45, 49 - ditto, retrolateral view; 46, 50 - epigyne, ventral view; 47, 51 - vulva, dorsal view. Scale bars: $(44,45,48,49) 0.2 \mathrm{~mm}$, $(46,47,50,51) 0.1 \mathrm{~mm}$.

Рис. 44-51. Копулятивные органы Carrhotus silanthi Caleb sp.n., голотип-самец и паратип-самка: 44, 48 - левая пальпа самца, снизу; 45, 49 - тоже, сбоку-сзади; 46, 50 - эпигина, снизу; 47, 51 - вульва, дорзально. Масштаб: $(44,45,48,49)$ 0,2 мм, (46, 47, 50, 51) 0,1 мм. 

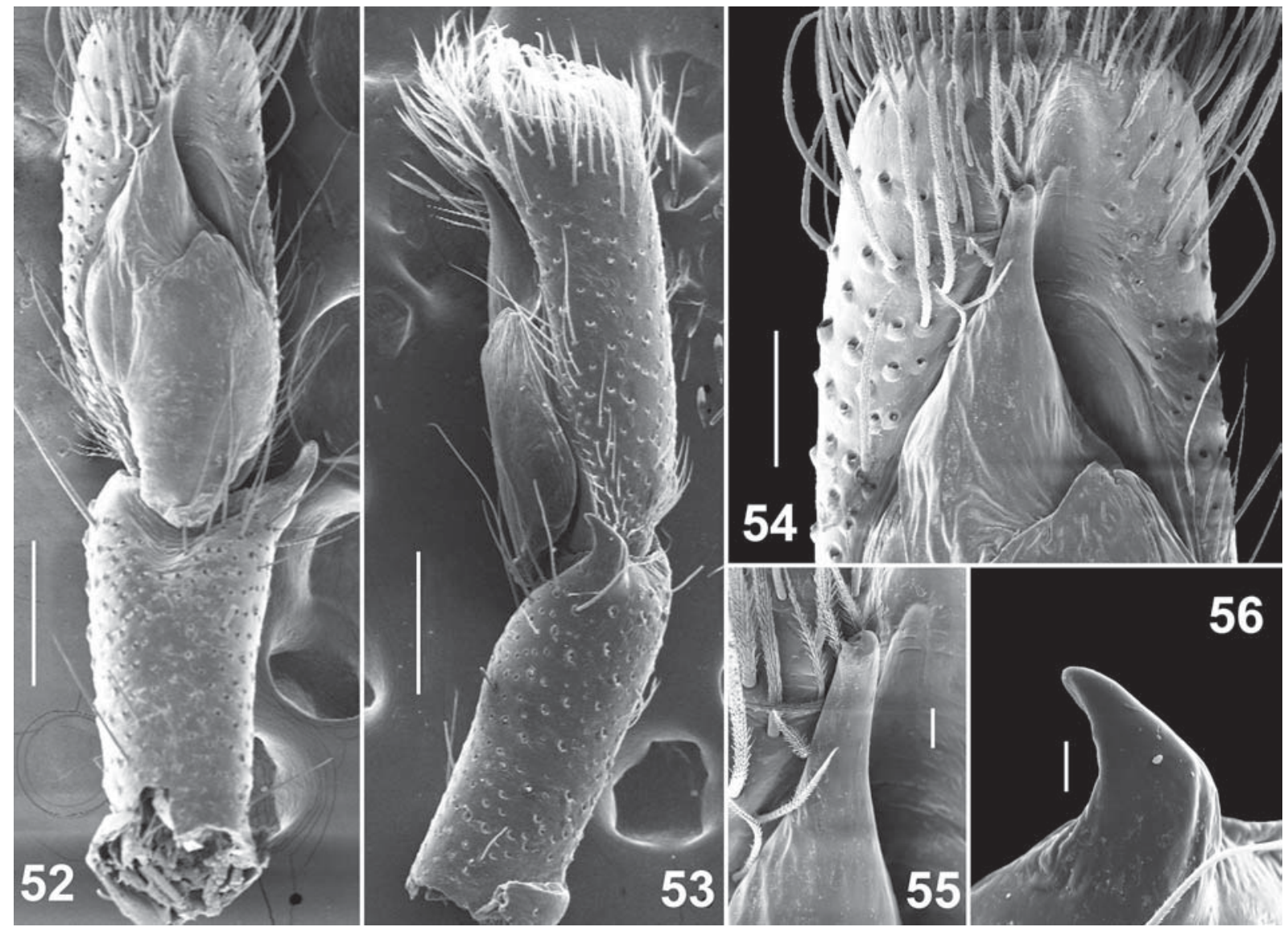

Figs 52-56. Left male palp of Carrhotus silanthi Caleb sp.n., holotype: 52 - ventral view; 53 - retrolateral view; 54, 55 - embolus; 56 - RTA, retrolateral view. Scale bars: $(55,56) 0.02 \mathrm{~mm},(54) 0.1 \mathrm{~mm},(52,53) 0.2 \mathrm{~mm}$.

Рис. 52-56. Левая пальпа самца Carrhotus silanthi Caleb sp.n., голотип: 52 - снизу; 53 - сзади-сбоку; 54, 55 - эмболюс; 56 отросток голени, сзади-сбоку. Масштаб: $(55,56) 0,02$ мм, (54) 0,1 мм, $(52,53) 0,2$ мм.

tibiae I 3036, II 3034, III 3133, IV 3133; metatarsi I 2024, II 2024, III 3044, IV 4044; tarsi I-IV 0000. Abdomen ovoid, densely covered with rusty brown hairs; anterior margin covered by white scales; pattern present on black background with a pair of white spots anteriorly and pairs of transverse stripes following posteriorly. Mid-dorsum covered with scales of metallic sheen (Figs 35, 36); venter yellowish, with a broad dark brown median region. Spinnerets brownish, covered with a patch of white hairs dorsally (Fig. 36). Palps dark brown; embolus short and thick with blunt tip; bulbus with posterior protrusion; RTA curved, claw-like (Figs 44, 45, 48, 49, 52-56).

Female (paratype). Total length 4.92 , carapace: 2.32 long, 1.83 wide; abdomen: 2.60 long, 1.91 wide. Eye measurements: AME 0.50, ALE 0.27, PME 0.08, PLE 0.22. AER 1.63, PER 1.74, EFL 1.03. Clypeus height 0.14. Leg measurements: I $4.25(1.36,0.81,0.86,0.70,0.52)$; II 3.98 $(1.34,0.79,0.73,0.64,0.48)$; III $4.32(1.49,0.75,0.76$, $0.80,0.52)$; IV $4.08(1.33,0.63,0.78,0.81,0.53)$. Leg formula: 3142. Leg spination: femora I 0700; II 0700, III 0700, IV 0700; patellae I-IV 1010; tibiae I 3036, II 3034, III 3133, IV 3133; metatarsi I 2024, II 2024, III 4034, IV 4043. Coloration pattern as in the male (Figs 39, 40), but differs as follows: AMEs surrounded by yellow orbital setae; clypeus covered with white hairs (Fig. 43); carapace with white hairs making an arc behind PLEs. Legs with white border on the distal margin of femur I-IV; other segments covered with sparse white hairs and black annulations alternatively. Epigyne with a pair of simple copulatory openings placed in small oval, yellowish depressions; insemination ducts short and diverge to join the stomach-shaped spermathecae (Figs 46, 47, 50, 51).

LIFE COLOUR. Both sexes look similar in general body colour pattern and are clothed with iridescent hairs. Colour varies from metallic green to bronze-green as shown in Fig. 40.

Carrhotus viduus (C.L. Koch, 1846)

Figs 57-83, Map 2.

Plexippus viduus C.L. Koch, 1846: 104, fig. 1166 (D $\left.{ }^{7}\right)$.

Carrhotus viduus: Andreeva et al., 1981: 103, figs 39-42 $\left(0^{7}+\right.$ ); Prószyński, 1992: 169, fig. 7 ( $\left.\bigcirc^{7}\right)$; Jastrzębski, 1999: 4, figs

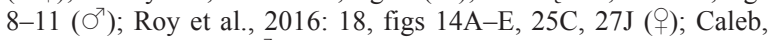
2016: 273 , figs $1-5\left(\mathrm{O}^{7}\right)$.

Marpissa decorata Tikader, 1974: 206, figs. 4-6 (Dㅇ); holotype \& paratype 9 in NZC-ZSI, examined; comb.n., syn.n.

Marpissa tikaderi Biswas, 1984: 125, figs 14-16 (Dㅇ); holotype 9 in NZC-ZSI, examined; comb.n., syn.n.

Marpissa lakshmikantapurensis Majumder, 2004: 32, figs 1115 (Do); holotype 9 in the NZC-ZSI, examined; comb.n., syn.n.

For a complete list of taxonomic references see WSC [2019]. 

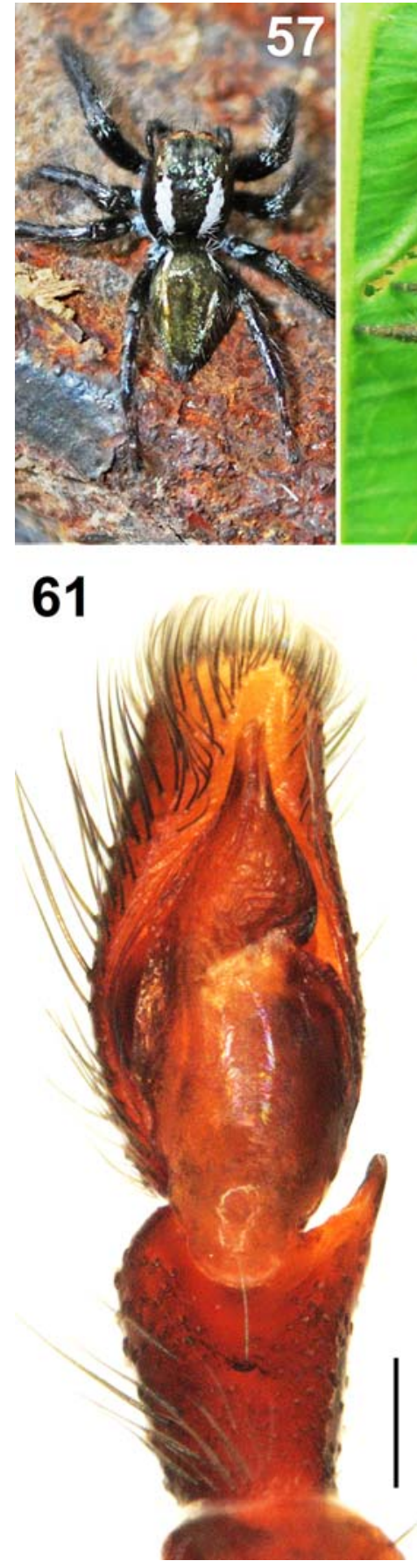
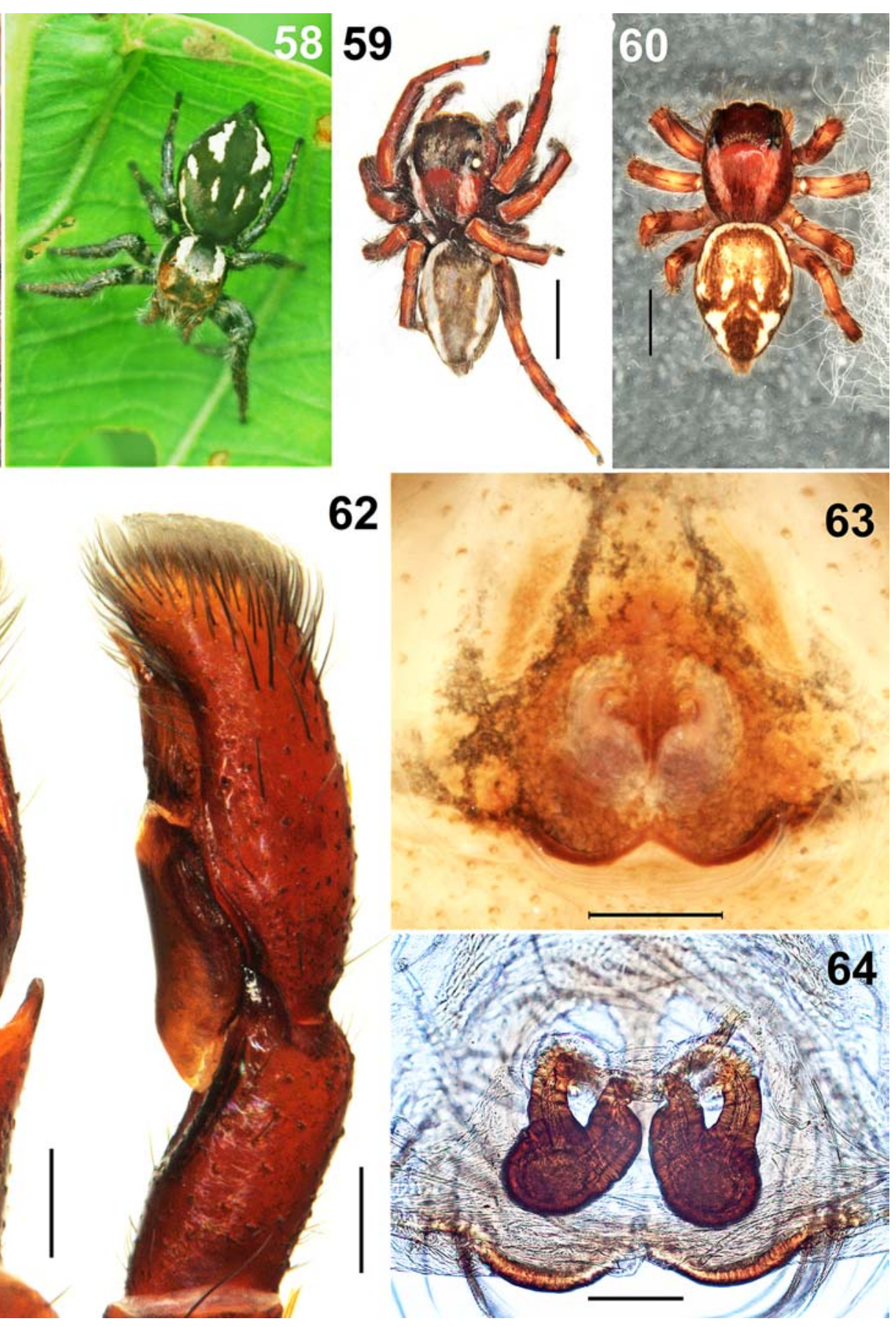

Figs 57-64. Somatic morphology and copulatory organs of Carrhotus viduus (C.L. Koch, 1846), specimens from Chennai (57, 58) and Andhra Pradesh (59-64): 57, 59 — male habitus, dorsal view; 58, 60 — female habitus, dorsal view; 61 - left male palp, ventral view; 62 — ditto, retrolateral view; 63 - epigyne, ventral view; 64 - vulva, dorsal view. Scale bars: (64) 0.1 mm, (61-63) 0.2 mm, (59, 60) 2 mm.

Рис. 57-64. Соматическая морфология и копулятивные органы Carrhotus viduus (C.L. Косh, 1846), экземпляры из Хенная (57, 58) и Андхра Прадеш (59-64): 57, 59 - габитус самца, сверху; 58, 60 - габитус самки, сверху; 61 - левая пальпа самца, снизу; 62 - тоже, сбоку-сзади; 63 - эпигина, снизу; 64 - вульва, сверху. Масштаб: (64) 0,1 мм, (61-63) 0,2 мм, (59, 60) 2 мм.

TYPES. Marpissa decorata Tikader, 1974: HoLOTYPE + (NZCZSI) from India, West Bengal, Calcutta, the Sibpur Botanical Garden, 10.11.1956, B.K. Tikader. Paratype: 1 o (NZC-ZSI), together with the holotype. - Marpissa lakshmikantapurensis Majumder, 2004: НоLотуPE 9 (NZC-ZSI 5470/18) from India, West Bengal, Lakshmikantapur, P.S. Mandirbazar, 18.11.1994, S.C. Majumder.
- Marpissa tikaderi Biswas, 1984: Holotype + (NZC-ZSI 5118/ 18) from India, West Bengal, Calcutta, Alipore, Zoological Garden, 04.01.1982, B. Biswas.

OTHER MATERIAL. INDIA: $1 \sigma^{\top}$ (NZC-ZSI 6259/18), West Bengal, North 24 Parganas Distr., Halisahar $\left(22.924791^{\circ} \mathrm{N}, 88\right.$. $\left.422547^{\circ} \mathrm{E}\right), 17 \mathrm{~m}$ a.s.1., 10.01.2017, S. Talukdar; $1 \sigma^{\top} \& 2$ sub- 

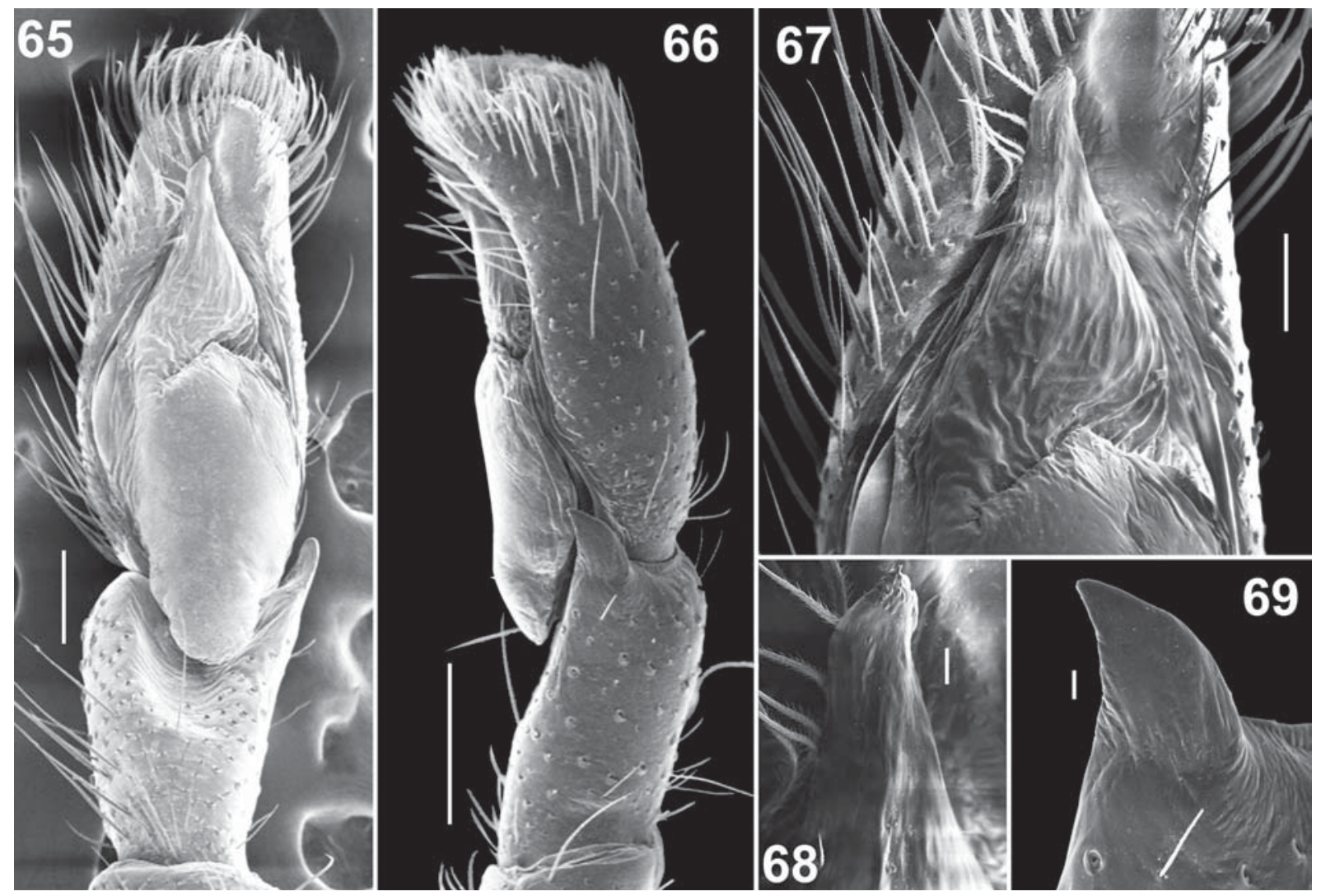

Figs 65-69. Left male palp of Carrhotus viduus (C.L. Koch, 1846): 65 - ventral view; 66 - retrolateral view; 67, 68 - embolus, ventral view; 69 - RTA, retrolateral view. Scale bars: $(68,69) 0.02 \mathrm{~mm},(67) 0.1 \mathrm{~mm},(65) 0.2,(66) 0.3 \mathrm{~mm}$.

Рис. 65-69. Левая пальпа самца Carrhotus viduus (C.L. Koch, 1846): 65 - снизу; 66 - сбоку-сзади; 67, 68 - эмболюс, снизу; 69 - тибиальный отросток, сбоку-сзади. Масштаб: $(68,69)$ 0,02 мм, (67) 0,1 мм, (65) 0.2, (66) 0,3 мм.

adults (NZC-ZSI 6313/18), same state and distr., Halisahar $\left(22.924791^{\circ} \mathrm{N}, 88.422547^{\circ} \mathrm{E}\right), 17 \mathrm{~m}$ a.s.1., 12.02 .2017 , S. Talukdar; $1 \mathrm{O}^{7}$ (NZC-ZSI 6361/18), Nagaland, Dimapur, Forest colony $\left(25.910434^{\circ} \mathrm{N}, 93.717406^{\circ} \mathrm{E}\right), 156 \mathrm{~m}$ a.s.l., 20.03 .2017 , Aneesh; 1 (NZC-ZSI $6454 / 18)$, Surat $\left(21.16^{\circ} \mathrm{N}, 72.83^{\circ} \mathrm{E}\right), 13.08 .1917$, unknown; $1 \sigma^{7}, 1$ ㅇ (NZC-ZSI 6462/18, 6463/18), West Bengal, South 24 Parganas Distr., Tentulberia, Garia Railway Station $\left(22.467030^{\circ} \mathrm{N}, 88.404348^{\circ} \mathrm{E}\right), 7 \mathrm{~m}$ a.s.1., 18.02 .2018 , C. Bera; $10^{7}$ (NZC-ZSI 6553/18), West Bengal, Nadia, Kalyani $\left(22.97^{\circ} \mathrm{N}\right.$, $\left.88.43^{\circ} \mathrm{E}\right), 13 \mathrm{~m}$ a.s.1., 17.09 .1969 , D. Sinharny; 1 \% (ZSI-CDTAA292), West Bengal, Sundarban Biosphere Reserve $\left(22.11^{\circ} \mathrm{N}\right.$, 88.84 ${ }^{\circ} \mathrm{E}$ ), $4 \mathrm{~m}$ a.s.1., 31.05.2016, Rushati Dey; $2 \mathrm{O}^{7} \mathrm{O}^{7}, 1$ ㅇ (NZCZSI 6916/18), Andhra Pradesh, Hope Island $\left(16.959111^{\circ} \mathrm{N}\right.$, $82.357583^{\circ} \mathrm{E}$ ), $5 \mathrm{~m}$ a.s.1., 27.03.2019, Rameshkumar. — IRAN (?): $20^{7} \sigma^{7}, 1$ + (NZC-ZSI 6674/18), "Hung, Persian, Beluchistan", W.T. Blanford.

COMMENTS. While examining old unidentified collections kept in the NZC-ZSI, we have identified three specimens of $C$. viduus collected by William Thomas Blanford (1832-1905) from "Beluchistan" (=Balochistan). An exact collecting locality in Balochistan is unknown. Perhaps, the specimens were collected during his appointment as a member of the Persian boundary commission (1870 to 1872), where he recorded that the bulk of his collection was obtained between Gwadar and Shiraz [Blanford, 1876]. This record represents the westernmost locality of the species distribution.

Three Indian species with identical colour patterns were described in the genus Marpissa C.L. Koch, 1846: viz., M. decorata Tikader, 1974, M. tikaderi Biswas, 1984 and M. lakshmikantapurensis Majumder, 2004. All of them were described solely from the females and from nearby locations in West Bengal (see inset of Map 2). M. decorata was first described from the Sibpur Botanical Garden, Kolkata, West Bengal and later was recorded from other localities in West Bengal, such as: Baikunthapur, Baraipur, Distr. 24 Parganas; Baranagar; Baishnabghata; Ballygunge Lake area [Tikader, Biswas, 1981]; Chapramari, Jalpaiguri [Sen et al., 2015]; Nepuchapur Tea Estate [Roy et al., 2016]. The holotype and paratype females of $M$. decorata were re-examined and found to be identical to C. viduus (cf. Figs 70-73 with Figs 60, 63, 64 and figs 41, 42 in Andreeva et al. [1981]). Thus, it is safe to conclude that the species $M$. decorata is to be treated as a member of Carrhotus and a junior synonym of $C$. viduus.

M. lakshmikantapurensis was described from Lakshmikantapur, South 24 Parganas Distr., West Bengal. The holotype female (Fig. 76) was apparently dissected and the copulatory organs kept in a small vial in another glass tube from which the epigyne was found missing. However, the male palp of $C$. viduus was found in the glass tube containing the vial (Figs 77, 78), probably belonging to the specimen collected together with the holotype female, indicating a potential matching of sexes and indicating the synonymy of both species names. Based on the general body morphology, the original illustrations of the copulatory organs and the aforementioned observation, it is safe to conclude that the species 

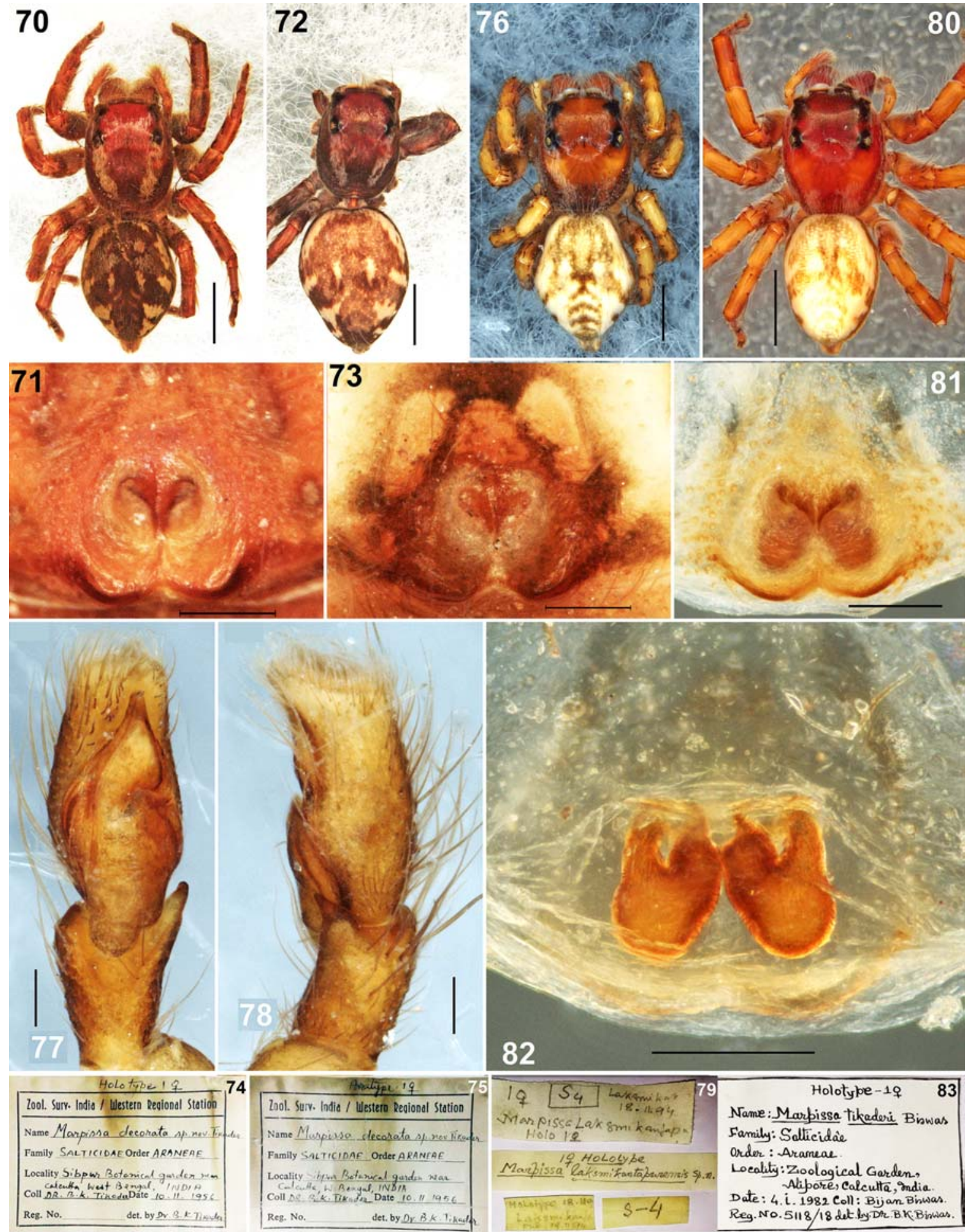

Figs 70-83. Somatic morphology and copulatory organs of Carrhotus viduus (C.L. Koch, 1846): the types of Marpissa decorata Tikader, 1974 (holotype female: 70, 71, 74 and paratype female: 72, 73, 75), the types of Marpissa lakshmikantapurensis Majumder, 2004 (holotype female: 76, 79 and the male palp found in the microvial: 77, 78) and holotype female of Marpissa tikaderi Biswas, 1984 (8083): 70, 72, 76, 80 - general appearance, dorsal view; 71, 73, 81 - epigyne, ventral view; 77 - male palp, ventral view; 78 - ditto, retrolateral view; 74, 75, 79, 83 - data labels; 82 - vulva, dorsal view. Scale bars: $(71,73,77,78,81,82) 0.2 \mathrm{~mm}$, $(70,72,76,80) 2 \mathrm{~mm}$.

Рис. 70-83. Соматическая морфология и копулятивные органы Carrhotus viduus (C.L. Koch, 1846): типы Marpissa decorata Tikader, 1974 (голотип-самка: 70, 71, 74 и паратип-самка: 72, 73, 75), типы Marpissa lakshmikantapurensis Majumder, 2004 (голотип-самка: 76, 79 и пальпа самца, найденная в микропробирке: 77, 78) и голотип-самка Marpissa tikaderi Biswas, 1984 (8083): 70, 72, 76, 80 - общий вид, сверху; 71, 73, 81 - эпигина, снизу; 77 - пальпа самца, снизу; 78 - тоже, сбоку-сзади; 74, 75, 79,83 - этикетки; 82 - вульва, сверху. Масштаб: $(71,73,77,78,81,82)$ 0,2 мм, $(70,72,76,80) 2$ мм. 


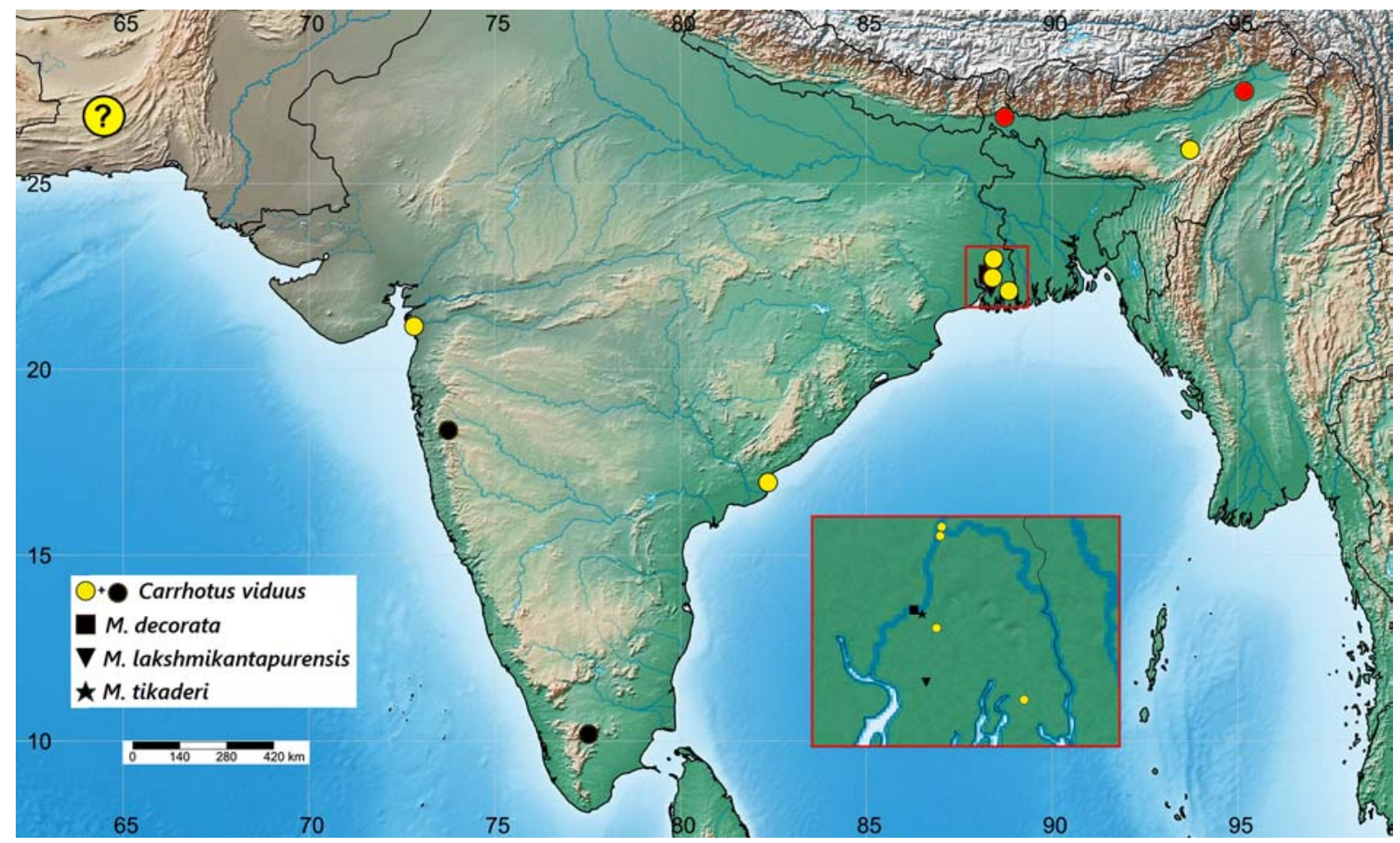

Map 2. Collecting localities of Carrhotus viduus in India. Yellow circles represent original records, black circles - those from the literature. Circle with ? mark represents the unknown locality from Balochistan. Inset shows the type localities of Marpissa species that were synonymized with $C$. viduus; explanations are given in the text.

Карта 2. Тоски находок Carrhotus viduus в Индии. Желтые кружки обозначают оригинальные находки, черные - литературные данные. Кружок с вопросительным знаком указывает на неизвестный локалитет в Белуджистане. Врезка показывает типовые локалитеты видов Marpissa, которые были синонисизированы с C. viduus; объяснения в тексте.

M. lakshmikantapurensis is to be treated as a member of Carrhotus and a junior synonym of $C$. viduus.

M. tikaderi was described from zoological garden in Alipore, Kolkata from a single female. The general morphology and copulatory organs (Figs 80-82) leave no doubt that this species is to be treated as a member of Carrhotus and a junior synonym of $C$. viduus (Figs 60, 63-64).

DISTRIBUTION. India to China [WSC, 2019], Balochistan (new record); the distribution in India is shown in Map 2.

\section{Carrhotus tristis Thorell, 1895 Map 1.}

COMMENTS. The species was originally described by Thorell [1895] from Myanmar. It was later redescribed from a male specimen from Kolkata, India [Prószyński, 1992]. The male palp with the characteristic long, filiform embolus (see figs 8-11 in Prószyński [1992]) indicates that this species seems not to be a member of Carrhotus. Its revised placement is to wait until both sexes have been collected together and described.

Carrhotus yunnanensis (Song, 1991) comb.n. Figs 18, 19.

Ptocasius yunnanensis Song, 1991: 165, figs. 3A-D (D+); the holotype in Institute of Zoology, Chinese Academy of Sciences, Beijing, China, not examined.
Carrhotus kevinlii Cao et Li, in Cao et al., 2016: 56, figs 9A$\mathrm{D}, 10 \mathrm{~A}-\mathrm{E}\left(\mathrm{D} \mathrm{O}^{\mathrm{T}}+\right.$ ); the types in Institute of Zoology, Chinese Academy of Sciences, Beijing, China, not examined, syn.n.

COMMENTS. Ptocasius yunnanensis was described from the holotype female from Menglun, Mengla County, Yunnan, China. Another species, Carrhotus kevinlii Cao et $\mathrm{Li}, 2016$ was recently described from the holotype male and four paratypes (two male and two female) taken from the close locality Xishunagbanna Nature Reserve situated in the same province Yunnan [Cao et al., 2016]. The females of the both aforementioned species are identical in their general body morphology, as well as in the conformation of their copulatory organs. The carapace with a pair of dark brown patches on the posterior slope; abdomen with mid-dorsal, chevron-shaped dark brown markings; the epigyne with slitshaped copulatory openings and strongly sclerotized edges and a pair of pockets along its posterior margin; insemination ducts short and broad; spermathecae globular with its diameter as wide as the copulatory ducts (cf. figs 3A,C,D in Song [1991] with figs 10A, B, D in Cao et al. [2016]). Based on these morphological observations and taking into account that both species occur in the same province, it is safe to conclude that the species $C$. kevinlii is conspecific to C. yunnanensis and its name is to be synonymized with the latter one.

Acknowledgements. We are grateful to Dr Kailash Chandra, Director of the Zoological Survey of India for the moral support and providing necessary facilities to carry out the work. We are especially thankful to Prof Dinendra Ray- 
chaudhuri of the Ramakrishna Mission Vivekananda University, Narendrapur, West Bengal for depositing the studied specimens in the NZC-ZSI; the specimens were collected as part of the All India Coordinated Project on Capacity Building in Taxonomy (AICOPTAX). He is also thanked for providing digital images of Bianor piratus. We thank Srabani Kayal for her kind assistance in obtaining SEM images. We thank the Forest Department officials of Andhra Pradesh and Nagaland States for their kind support. John Caleb thanks the American Arachnological Society for an Arachnological research grant from the Herbert Levi fund (HLMFAR). Finally, our sincere thanks go to Dr Dmitri Logunov (Manchester, UK) for the constructive inputs to the $\mathrm{ms}$, helping us to improve it greatly.

\section{References}

Andreeva E.M., Kononenko A.P., Prószyñski J. 1981. Remarks on genus Mogrus Simon, 1882 (Aranei, Salticidae) // Annales Zoologici, Warszawa. Vol.36. P.85-104.

Blanford W.T. 1876. Eastern Persia: An account of the journeys of the Persian Boundary Commission, 1870-71-72. The Zoology and Geology. London. Vol.2. P.1-516.

Biswas B. 1984. Description of six new species of spiders of the families Clubionidae, Gnaphosidae and Salticidae from India // Bulletin of the Zoological Survey of India. Vol.6. P.119-127.

Caleb J.T.D. 2016. New data on the jumping spiders (Araneae: Salticidae) from India // Arthropoda Selecta. Vol.25. No.3. P. 271-277.

Cao Q., Li S.Q., Żabka M. 2016. The jumping spiders from Xishuangbanna, Yunnan, China (Araneae, Salticidae) // ZooKeys. Vol.630. P.43-104.

Dhali D.C., Saha S., Raychaudhuri D. 2017. Litter and ground dwelling spiders (Araneae: Arachnida) of reserve forests of Dooars, West Bengal // World Scientific News. Vol.63. P.1242.

Jastrzębski P. 1999. Salticidae from the Himalaya: The genus Carrhotus Thorell 1891 (Araneae, Salticidae) // Senckenbergiana Biologica. Vol.79. P.1-9.

Koch C.L. 1846. Die Arachniden. Nürnberg: J.L. Lotzbeck. Bd.13, $234 \mathrm{~S}, \mathrm{Bd} .14,88 \mathrm{~S}$

Maddison W.P. 2015. A phylogenetic classification of jumping spiders (Araneae: Salticidae) // Journal of Arachnology. Vol.43. No.3. P.231-292.

Majumder S. C. 2004. Taxonomic studies of some spiders from mangrove and semi-mangrove areas of Sundarban // Memoirs of the Zoological Survey of India. Vol.20. No.2. P.1-42.

Montesanto G. 2015. A fast GNU method to draw accurate scientific illustrations for taxonomy // Taiti S., Hornung E., Štrus J.,
Bouchon D. (eds.). Trends in Terrestrial Isopod Biology. ZooKeys. Vol.515. P.191-206.

Prószyński J. 1976. Studium systematyczno-zoogeograflczne nad rodziną Salticidae (Aranei) Regionów Palearktycznego i Nearktycznego. Wyższa Szkola Pedagogiczna v Siedlcach. 260 p.

Prószyński J. 1992. Salticidae (Araneae) of India in the collection of the Hungarian National Natural History Museum in Budapest // Annales Zoologici, Warszawa. Vol.44. P.165-277.

Prószyński J. 2017. Pragmatic classification of the world's Salticidae (Araneae) // Ecologica Montenegrina. Vol.12. P.1-133.

Roy T.K., Saha S., Raychaudhuri D. 2016. A treatise on the jumping spiders (Araneae: Salticidae) of tea ecosystem of Dooars, West Bengal, India // World Scientific News. Vol.53. No.1. P.1-66.

Saaristo M.I. 2010. Araneae // Gerlach J., Marusik Y.M. (eds.). Arachnida and Myriapoda of the Seychelles islands. Manchester UK: Siri Scientific Press. P.8-306.

Sen S., Dhali D.C., Saha S., Raychaudhuri D. 2015. Spiders (Araneae: Arachnida) of Reserve Forests of Dooars: Gorumara National Park, Chapramari Wildlife Sanctuary and Mahananda Wildlife Sanctuary // World Scientific News. Vol.20. P.1-339.

Shorthouse D.P. 2010 SimpleMappr, an online tool to produce publication-quality point maps. Available from http:// www.simplemappr.net (accessed 11 July 2019).

Song D.X. 1991. Three new species of the genus Ptocasius from China (Araneae: Salticidae) // Sinozoologia. Vol.8. No.163168.

Thorell T. 1877. Studi sui Ragni Malesi e Papuani. I. Ragni di Selebes raccolti nel 1874 dal Dott. O. Beccari // Annali del Museo Civico di Storia Naturale di Genova. Vol.10. P.341637.

Thorell T. 1891. Spindlar från Nikobarerna och andra delar af södra Asien // Kongliga Svenska Vetenskaps-Akademiens Handlingar. Bd.24. H.2. S.1-149.

Thorell T. 1895. Descriptive catalogue of the spiders of Burma. London. 406 p.

Tikader B.K. 1974. Studies on some jumping spiders of the genus Marpissa from India (family-Salticidae) // Proceedings of the Indian Academy of Science. Vol.79. No.B. P.204-215.

Tikader B.K., Biswas B. 1981. Spider fauna of Calcutta and vicinity: Part-I // Records of the Zoological Survey of India, Occasional Paper. Vol.30. P.1-149.

WSC 2019. World Spider Catalog. Version 20.0. Natural History Museum Bern, online at http://wsc.nmbe.ch, accessed on 17 September 2019. doi: 10.24436/2.

Żabka M. 1985. Systematic and zoogeographic study on the family Salticidae (Araneae) from Viet-Nam // Annales Zoologici, Warszawa. Vol.39. P.197-485.

Responsible editor D.V. Logunov 